CYSTIC FIBROSIS

\title{
A survey of sexual and reproductive health in men with cystic fibrosis: new challenges for adolescent and adult services
}

\author{
S M Sawyer, B Farrant, B Cerritelli, J Wilson
}

Thorax 2005;60:326-330. doi: 10.1136/thx.2004.027599

See end of article for authors' affiliations

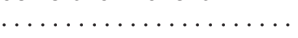

Correspondence to: Professor S M Sawyer, Centre for Adolescent Health, 2 Gatehouse Street, Parkville 3052, Victoria, Australia; Susan sawyer@rch.org.au

Received 30 April 2004 Accepted 6 January 2005

\begin{abstract}
Background: Improved survival dramatically alters the consequences of adult co-morbidities in men with cystic fibrosis (CF) such as male infertility. Few studies have systematically addressed the impact of sexual and reproductive health issues in these men or considered the implications for healthcare delivery.

Method: A descriptive cohort study was undertaken using a sexual and reproductive health survey of men from a large adult CF centre, including men with lung transplantation.

Results: The mean (SD) age of the 94 men (response rate $75 \%$ ) was $30.5(7.6)$ years. $94 \%$ knew that men with CF had reduced fertility. Men first heard about infertility later than desired $(p<0.001)$ and only 53\% heard from their preferred source. Men who were told about infertility when older were more likely to be upset than those told earlier ( $p<0.01)$. 53\% of men had undergone semen analysis: $68 \%$ of men who had not been tested wanted semen analysis. $73 \%$ believed semen analysis should occur before 18, but the youngest age of testing was 24 years. In adolescence, one in three men had assumed they did not need to use condoms and one in 10 had confused infertility with impotence. $66 \%$ of men wanted more information on reproductive options and $84 \%$ wanted children. Seventeen men were parents by natural conception $(n=1)$, micro-epididymal sperm aspiration $(n=6)$, donor sperm $(n=9)$, and through step children $(n=1)$. Conclusions: Men with CF desire more sexual and reproductive health information. Earlier discussion of sexual and reproductive health is indicated in paediatric settings, and semen analysis should be routinely offered. In adult services greater discussion of reproductive health options is indicated.
\end{abstract}

$\mathrm{T}$ here is now an emerging understanding of the importance of men's health when compared with the well established notion of women's health. ${ }^{1}$ Discussion of men's health is commonly framed as men's unwillingness to look after themselves, with reference to the "absent male" within healthcare settings. ${ }^{2}$ Certainly men are less likely to seek preventive health care. ${ }^{3}$ However, it is also important to consider how healthcare services can best promote men's health, including their sexual and reproductive health. This is relevant for both speciality and primary health care, especially when caring for men with chronic disorders, diseases or conditions that affect sexual and reproductive health such as HIV, depression, diabetes, and cystic fibrosis (CF).

CF is one of the few disorders where male infertility is expected. It was first described in the late $1960 \mathrm{~s}^{4-6}$ at a time when only $50 \%$ of patients survived to 12 years. ${ }^{7}$ Thirty five years later, survival into the third decade is now expected, ${ }^{8}$ which has dramatically altered the significance of infertility. 95-98\% of men with CF are azoospermic. The CF genotype is responsible for aberrant development of the reproductive portion of the mesonephric duct leading to bilateral absence of the vas deferens. Dysfunction and/or absence of the seminal vesicles accounts for low volume ejaculate. ${ }^{9}$ Active spermatogenesis occurs within the testis and sexual function is not affected. A minority of men are fertile, especially those with the 3849-10 kb C-T mutation, ${ }^{10}{ }^{11}$ highlighting the role of semen analysis. Recent developments in reproductive technologies using microsurgical epididymal sperm aspiration and intracytoplasmic sperm injection have facilitated men with bilateral absence of the vas deferens to father children. ${ }^{9}$ Conception rates vary between $30 \%{ }^{9}$ and $35 \%{ }^{12}$ per cycle, achieving pregnancy in $62.5 \%$ of couples where the man has CF. ${ }^{12}$
Apart from infertility, few studies have addressed the sexual and reproductive health needs of men with $\mathrm{CF}$. The earliest studies, ${ }^{13}{ }^{14}$ undertaken 20 years after the initial reports of male infertility, identified that men with CF were strikingly uninformed about the sexual and reproductive health complications of CF. Thirty years after these initial reports, increasing awareness of infertility was shown in studies in Boston, ${ }^{15}$ Birmingham, ${ }^{16}$ and Scotland. ${ }^{17}$ However, these studies also showed that men with CF were relatively uninformed about many broader aspects of reproductive and sexual health such as semen analysis, assisted reproductive technologies, or prevention of sexually transmitted diseases, and that they consistently desired more information. ${ }^{15-17}$

The small number of studies limits the capacity to interpret apparent differences. For example, Fair et al ${ }^{17}$ found that two thirds of Scottish men reported negative emotions such as shock and bewilderment when told of infertility. In contrast, Sawyer $e$ a $l^{15}$ found that $90 \%$ of US men who were told about infertility in adolescence were not overly distressed at the time. This could be explained by a range of factors, such as the context in which men were told, the person who told them, and the age they were told. It could also reflect cultural differences between the different study populations, as it is not known how representative the few published studies are of other populations of men with $\mathrm{CF}$ - and the practices of their healthcare providers.

A large number of questions remain unanswered. What is the appropriate age to first discuss male infertility? What is the effect of hearing about infertility at different ages? What is the value of confirming fertility status by semen analysis? Who should discuss sexual and reproductive health and what should be discussed? Similarly, it is not known what proportion of adult men with CF are informed about assisted reproductive technologies and wish to use them. No studies 
have included men with lung transplantation, whose views may or may not differ from those without lung transplantation. Understanding these aspects of male sexual and reproductive health would greatly inform clinical practice in both adolescent as well as adult men with CF, with implications for healthcare services more broadly.

In this study we aimed to address these questions in detail by assessing the knowledge, attitudes, and behaviours in relation to sexual and reproductive health in a large representative group of Australian men with CF.

\section{METHODS}

\section{Participants}

Eligible men attended the CF Clinic at the Alfred Hospital, Melbourne, Victoria, the regional adult CF tertiary service in Victoria which manages 232 non-transplanted and 32 transplanted men and women. Men receiving palliative care were not eligible for the study. Ethical approval was obtained from the Alfred Hospital ethics committee.

\section{Questionnaire}

A written questionnaire was developed from previous qualitative work. ${ }^{15}$ The 40 questions took about 20 minutes to complete. Questions included aspects of knowledge, attitudes, and behaviour in relation to sexual and reproductive health. Men were asked to describe their health status as mild, moderate or severe (self-report) and the mean percentage predicted forced expiratory volume in 1 second $\left(\mathrm{FEV}_{1} \%\right)$ for the year before the survey was recorded.

\section{Data collection}

Eligible subjects were mailed an information sheet, consent form and questionnaire to be returned by mail. Nonresponders received a telephone reminder and were also reminded at their next clinic visit.

\section{Analysis of data}

Data were entered into Epidata, then transferred to STATA Version 8.2 for analysis (Stata Corporation, Texas, USA) which was predominantly descriptive. The results are presented as percentages or median (range) as appropriate. Comparison between groups was made using the $\chi^{2}$ test for categorical variables (Fisher's exact test for small samples) and, for continuous variables, the $t$ test for parametric data and Wilcoxon signed rank test for non-parametric data. Paired $t$ tests (for parametric data) and the Mann-Whitney U test (for non-parametric data) were used for paired comparisons.

\section{RESULTS}

\section{Response rate}

Questionnaires were mailed to 131 subjects, of whom 12 chose not to participate and 19 did not return their questionnaires. Six were retrospectively considered ineligible due to terminal illness $(\mathrm{n}=1)$; uncontactable $(\mathrm{n}=3)$; and deceased $(n=2)$. Thus, 94 questionnaires were returned from the eligible 125 , resulting in a response rate of $75 \%$. The response rate from the 18 transplanted subjects was $89 \%$ compared with $72 \%$ from the 109 non-transplanted subjects.

\section{Demographic data}

The mean (SD) age of the participants was 30.5 (7.6) years (range 18-54). Most were in a relationship and were employed (table 1).

Seventeen percent of the subjects had undergone lung transplantation. The transplanted group was not significantly different from the non-transplanted group in age, marital status, or employment status. The only significantly different variable was self-rating of disease severity, with more
Table 1 Demographic information on study participants

\begin{tabular}{|c|c|}
\hline & n (\%) \\
\hline \multicolumn{2}{|l|}{ Relationship status ( $\mathrm{n}=94$ ) } \\
\hline Single, not in relationship & $34(36)$ \\
\hline Single, in relationship & $19(20)$ \\
\hline De facto/married & $39(42)$ \\
\hline Separated/divorced & $2(2)$ \\
\hline \multicolumn{2}{|l|}{ Employment status $(n=94)$} \\
\hline Employed (full/part time) & $65(69)$ \\
\hline Student (full/part time) & $8(9)$ \\
\hline Home maker & $2(2)$ \\
\hline Unemployed & $19(20)$ \\
\hline Heart/lung transplant & $16(17)$ \\
\hline \multicolumn{2}{|c|}{ Self-reported disease severity $(n=91)$} \\
\hline Mild & $41(44)$ \\
\hline Moderate & $33(35)$ \\
\hline Severe & $17(18)$ \\
\hline \multicolumn{2}{|c|}{ Pulmonary function (non-transplant, $n=85$ ) } \\
\hline Mild $\left(\mathrm{FEV}_{1} \geqslant 75\right)$ & $22(26)$ \\
\hline Moderate $\left(45 \leqslant \mathrm{FEV}_{1}<75\right)$ & $25(29)$ \\
\hline Severe $\left(F E V_{1}<45\right)$ & $38(45)$ \\
\hline
\end{tabular}

transplanted subjects rating their disease as severe $(\mathrm{p}<0.05)$. We therefore analysed transplanted and nontransplanted subjects together.

The mean $\mathrm{FEV}_{1}$ for non-transplanted subjects was 53\% (range 17-108) compared with 62\% (range 26-123) for transplanted subjects. There was no difference in lung function between study participants and non-responders, with a mean $\mathrm{FEV}_{1}$ in non-responders of 47\% (range 18-77). There was fair agreement between self-reported disease status and $\mathrm{FEV}_{1} \%$ (kappa $=0.31,95 \%$ CI 0.19 to 0.48), although self-rating tended to underestimate disease severity.

\section{Fertility knowledge}

Only one participant (aged 19 years) was unaware that CF commonly affects male fertility. While nearly three quarters of the remaining 93 men believed that men with CF were nearly always infertile (table 2), 25\% were incorrect or uncertain about the effect of CF on fertility.

The mean (SD) age at which men reported they were first told anything about CF and infertility was 16.4 (4.1) years (range 7-31). This was significantly later $(p<0.001)$ than the mean age that men reported they would like to be told (14.4 (2.8) years, range 6-24). A third of men (32\%) reported they had been told about fertility problems before they were 15 years old.

Men reported first hearing about male fertility problems in CF from a variety of sources, most commonly from CF clinic staff (table 3). One of the men reported first hearing about male infertility from a medical student, while another learnt about it during his own university studies. 53\% of the men

\begin{tabular}{lc}
$\begin{array}{l}\text { Table } 2 \\
(\mathrm{n}=93)\end{array}$ & Knowledge of fertility in men with CF \\
\hline & $\mathrm{n}(\%)$ \\
\hline How does CF affect fertility in men? & $2(2)$ \\
Normal fertility & $15(16)$ \\
Reduced fertility & $72(77)$ \\
Nearly always infertile & $4(4)$ \\
Don't know & $71(76)$ \\
Why does CF affect male fertility? & $12(13)$ \\
Transport problem & $2(2)$ \\
Sperm production problem & $9(10)$ \\
Transport and sperm production problem & \\
Don't know & \\
\hline
\end{tabular}


Table 3 Sources of initial $(n=92)$ and preferred $(n=93)$ information about fertility problems

\begin{tabular}{|c|c|c|c|}
\hline $\begin{array}{l}\text { From whom did you first hear about } \\
\text { male fertility problems? }\end{array}$ & n (\%) & $\begin{array}{l}\text { From whom would you like to have heard } \\
\text { first about male fertility problems? }\end{array}$ & n (\%) \\
\hline CF clinic staff & $51(55)$ & CF clinic staff & $46(49)$ \\
\hline Parents & $12(13)$ & Parents & $27(29)$ \\
\hline Other CF patients/friends & $10(11)$ & Other CF patients/friends & $1(1)$ \\
\hline Written material & $13(14)$ & Written material & $5(5)$ \\
\hline $\begin{array}{l}\text { Combination of clinic staff, parents, } \\
\text { other patients and written }\end{array}$ & $4(4)$ & $\begin{array}{l}\text { Combination of clinic staff, parents, } \\
\text { other patients and written }\end{array}$ & $14(15)$ \\
\hline Other & $2(2)$ & Other & 0 \\
\hline
\end{tabular}

reported they first heard about fertility problems from their preferred source. Men who reported first hearing about fertility problems from their parents were told at a significantly younger age (mean 13.2 years, range $7-16$ ) than men who were first told by CF clinic staff (mean 17.2 years, range $13-31 ; \mathrm{p}<0.001)$.

Half the subjects $(51 \%)$ reported no major reaction when first told about fertility problems in CF, 32\% were a little upset and $18 \%$ were very upset. Men who were told when they were older were more likely to report being upset than those told earlier $(\mathrm{p}<0.01$, fig 1$)$. There was no difference in reaction between subjects aged under 16 and those aged 1620 years when first told, although there was a trend towards a more negative reaction to being told in those men who reported first hearing about fertility problems from a nonpreferred source $(\mathrm{p}=0.09)$.

Thirty nine men (42\%) reported that the impact of fertility problems had become greater over time. Only 21 reported less impact over time. Men who first heard about fertility problems when aged less than 20 years were more likely to report that the significance of infertility had increased over time ( $p=0.04$, fig 2 ). The significance of fertility problems was reported to increase over time in all age groups except men currently aged over 40 years. Men who wanted children were more likely to report that the significance of infertility had increased with time $(\mathrm{p}<0.05)$.

\section{Semen analysis}

Forty nine men (53\%) reported that semen analysis had been undertaken, with 24 years being the youngest age at testing. Semen analysis was more likely in men who were older and married $(\mathrm{p}<0.001)$.

Men were asked to nominate the most appropriate age to be offered semen analysis: $73 \%$ reported $17-18$ years, $22 \%$ preferred $19-20$ years, and 5\% preferred over 20 years. Two thirds $(68 \%)$ of the 38 who had not had semen analysis reported that they wanted fertility testing: these men were

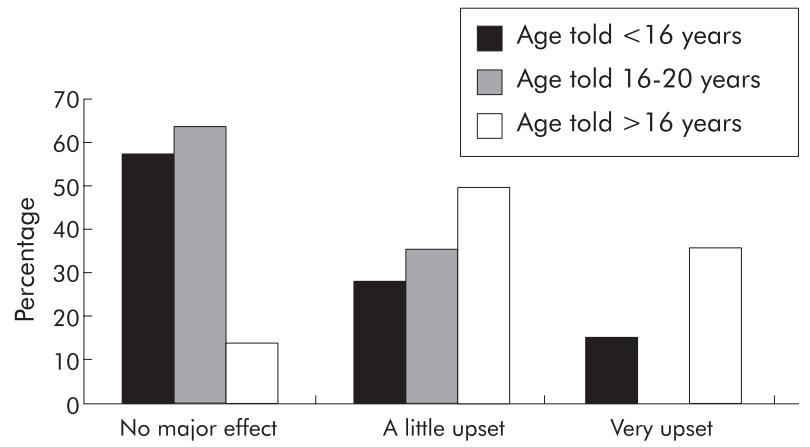

Figure 1 Reaction to first hearing about infertility according to age.

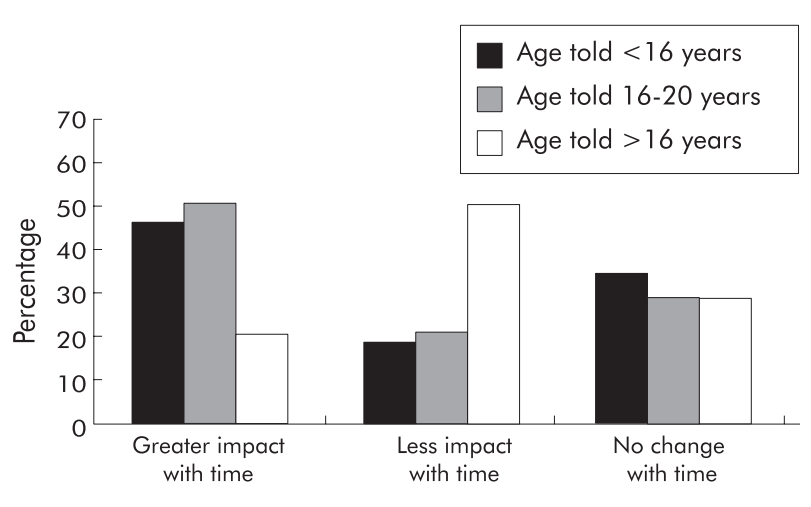

Figure 2 Impact of infertility knowledge over time.

more likely to be in a relationship $(\mathrm{p}<0.05)$ and more likely to want children in the future $(\mathrm{p}<0.05)$.

\section{Sexual function}

Ninety six percent of men had been sexually active. The mean (SD) age of first sexual intercourse was 17.9 (3.5) years (range 10-32); 38\% reported never having had a nocturnal emission, $44 \%$ reported they had had occasional nocturnal emissions, while $2 \%$ reported frequent emissions. A further 15 were unable to remember.

Nearly one in 10 men (9\%) reported confusing infertility with impotence as an adolescent. However, nearly one in three men (30\%) reported they had assumed that they did not need to use a condom as an adolescent. Men who had first been told about fertility problems by a healthcare professional were more likely to assume that they did not need to use condoms than those who had first been told by parents or friends $(\mathrm{p}<0.05)$.

\section{Effect on relationships}

Over one in five men (22\%) believed that infertility had affected their personal relationships. There was no significant association between an effect on relationships with age or marital status, whether they had children or wanted children, or their self-reported health status.

\section{Reproduction}

One in three men $(66 \%)$ reported wanting more information on reproductive options. These 62 men identified a variety of preferred sources of information, often multiple, including the CF team $(n=19)$, specialist reproductive experts $(\mathrm{n}=30)$, and written information $(\mathrm{n}=34)$. Those who wanted more information about male reproduction or reproductive options were more likely to be in a relationship but not married $(\mathrm{p}<0.01)$, to want children $(\mathrm{p}<0.001)$, and to want to know their fertility status $(\mathrm{p}<0.01)$. 
Seventeen of the men (18\%) had a total of 27 children (range 1-4). Seven of these 17 men were the biological fathers: six were conceived through assisted reproduction and one was conceived naturally. Of the remaining 10, nine had used artificial insemination by donor sperm and one had stepchildren. Men with mild disease were more likely to be fathers $(p<0.05)$. Eight of these 17 men wanted more children.

A high proportion of men without children (84\%) reported wanting children in the future. These men were no more likely to have had semen analysis but were more likely to want it $(\mathrm{p}<0.05)$. Disease severity, whether self-rated or $\mathrm{FEV}_{1} \%$, did not relate to a desire to have children.

\section{DISCUSSION}

The widespread understanding of infertility in CF in this large cohort of adult men is reassuring and supports a trend towards improved knowledge of male infertility over time. ${ }^{13-17}$ However, there continues to be a discrepancy between when men first hear and when they wish to hear about infertility. ${ }^{15}{ }^{17} \mathrm{~A}$ key finding is the apparent deficiencies in service delivery in relationship to male sexual and reproductive health that have not previously been described.

This is the first study of men with CF that has investigated the role of semen analysis in detail. We showed a higher rate of semen analysis (53\%) than in previous studies ${ }^{15}{ }^{16}$ which had rates of $31 \%$ and $24 \%$, respectively. However, a striking finding was that, while $95 \%$ of the men in this study felt that semen analysis should be performed before the age of 20, the youngest age of semen analysis was reported to be 24 years. An equally salient finding was that $68 \%$ of men who had not been tested wanted semen analysis, independent of their relationship status, suggesting that semen analysis should at the very least be offered to all men. The preferred age of testing has significant health service implications, especially for paediatric services.

This study identified that men's reaction to hearing about infertility varies with age. Thos who are told at a younger age are less likely to have a negative reaction at the time but are more likely to report a change in significance over time, especially if they want children. This reinforces earlier qualitative work $^{15}$ and emphasises the importance of continuing to discuss reproductive health issues over time.

Another key finding warranting greater attention is men's desire for more information about reproductive options, expressed by two out of three men. Advances in reproductive technology now make it possible for men with CF to father children. Indeed, almost one in five men in this study were parents, higher than the previously reported rates of $1-8 \%{ }^{15-17}$ Very high interest in future parenting was expressed by the men in this study, with $78 \%$ wanting children regardless of disease severity.

Consistent with previous studies, ${ }^{15} 17$ men in this study reported first hearing about male infertility from a variety of sources. It remains a concern that only half the men heard from what they considered to be their preferred source. The question of who should lead these discussions with boys is becoming clearer over time. As in the study by Madge and Carr, ${ }^{18}$ our results highlight that men with CF report wanting discussions with both CF clinic staff and parents; they do not want to hear from other patients or friends. Written information appears a valuable adjunct, especially in relation to reproductive options for adult men.

As in other studies, ${ }^{13-15}$ CF healthcare providers are identified as an important source of information about sexual and reproductive health. In this study CF clinic staff were most commonly preferred as the source of sexual and reproductive health information in adolescence. How well do clinic staff handle this responsibility? In terms of timing, it is concerning that CF health professionals discussed sexual and reproductive health issues significantly later than did parents, and later than desired by young people themselves. The majority of men believe these discussions should start when boys are less than 16 years old, the responsibility of paediatric or adolescent services. Adolescent boys enter puberty approximately 1 year later than girls, but there is no evidence from this study that discussions about sexual and reproductive health should occur later in boys with CF than girls. ${ }^{19}$

It has previously been documented that sexual and reproductive health discussions are generally initiated by boys or men rather than healthcare providers. ${ }^{17}{ }^{20} \mathrm{Few}$ men with CF feel comfortable initiating these discussions, ${ }^{17}$ reinforcing the importance of health professionals to take the lead in initiating discussions about sexual and reproductive health. The embarrassment and lack of training expressed by CF healthcare providers about these discussions $^{21}$ reinforces the importance of education and training about sexual health for respiratory health professionals.

What of content? The finding that men who had been told about fertility problems by health professionals were more likely to assume they did not need to use condoms is concerning and questions the messages young men are both given and receive. There are no formal guidelines for physicians about discussions of sexual and reproductive health care in men with CF against which we can compare our results, although a number of recommendations have been made. ${ }^{15-23}$ For example, Lyon and Bilton ${ }^{22}$ suggest seven points of information about infertility for men with CF: that $95 \%$ of men with CF are infertile; that infertility does not mean impotence; that spermatogenesis is likely to be normal; that ejaculatory fluid will not contain sperm because of bilateral absence of the vas deferens; that ejaculatory fluid is of low volume; that sperm aspiration and intracytoplasmic sperm injection can result in successful pregnancy; and that genetic counselling is available. Our study suggests further emphasis is warranted around infertility, semen analysis, and the prevention of sexually transmitted infections in the paediatric setting, with a greater focus on reproductive options within adult healthcare services.

One strength of this study is that it provides detailed information in an area where there are conspicuously few contemporary studies. ${ }^{15-17}$ Furthermore, it was conducted in a large CF centre with a good response rate. It is the first study to include a significant number of men with lung transplants. The absence of other studies means we cannot be clear how representative these findings are of other Australian or international centres. This study retrospectively sought information from adult men about events from their adolescence, a limitation common to most studies in this area $^{16-17}$ Indeed, there is only one qualitative study that addresses sexual and reproductive health directly with adolescent boys. ${ }^{15}$ This is an important opportunity for future research, as is the need to better understand parent perspectives of adolescent sexual and reproductive health. A recent study comparing mother and daughter perspectives about the timing and content of sexual and reproductive health discussions in girls with $\mathrm{CF}^{19}{ }^{24}$ highlighted the opportunities of greater maternal involvement. There is every reason to think that this is also relevant for boys with $\mathrm{CF}$ and their parents.

Finally, there are significant ethical issues about reproduction in men with CF that warrant further research. The apparent paradox of promoting population screening to reduce the burden of CF disease while supporting men with CF to parent children who will be carriers has already been noted. ${ }^{22}$ More practically, however, little is known about the decision making process of men with $\mathrm{CF}$ and their partners in 
balancing their medical needs and shortened life span with the desire to parent children. Further ethical issues arise in relation to partner screening in terms of understanding how couples would view having a child with $\mathrm{CF}$, as well as the role of selective termination of affected fetuses. Also, the issue of mispaternity must be recognised when undertaking semen analysis in men and genetic testing of children.

In contrast to the expressed concerns of men's lack of interest in their health, ${ }^{1-3}$ the men in this study appeared greatly interested in their sexual and reproductive health. We have shown that the relative importance of different issues changes over time. This study highlights the opportunities for both paediatric and adult CF healthcare services to better address male reproductive and sexual health.

\section{ACKNOWLEDGEMENT}

The authors acknowledge the contribution of Dr Catherine Bennett, School of Population Health, The University of Melbourne.

\author{
Authors' affiliations \\ S M Sawyer, B Farrant, B Cerritelli, Centre for Adolescent Health, \\ Melbourne, Australia \\ S M Sawyer, B Cerritelli, Department of Respiratory Medicine, Royal \\ Children's Hospital, Melbourne, Australia \\ S M Sawyer, Department of Paediatrics, University of Melbourne, \\ Melbourne, Australia \\ J Wilson, Department of Respiratory Medicine, The Alfred Hospital, \\ Melbourne, Australia

\section{REFERENCES} \\ 1 Courtenay WH. Constructions of masculinity and their influence on \\ men's well-being: a theory of gender and health. Soc Sci Med \\ 2000;50:1385-401. \\ 2 Baker P. The international men's health movement. BMJ 2001;323:1014-5. \\ 3 Hall RH. Promoting men's health. Aust Fam Physician 2003;32:401-7.
}

4 Denning CR, Sommers SC, Quigley HJ. Infertility in male patients with cystic fibrosis. Pediatrics 1968;41:7-17.

5 Kaplan E, Shwachman H, Perlmutter AD, et al. Reproductive failure in males with cystic fibrosis. N Engl J Med 1968;279:65-9.

6 Landing BH, Wells TR, Wang C-I. Abnormality of the epididymis and vas deferens in cystic fibrosis. Arch Pathol 1969;88:569-80.

7 Phelan PD, Allan JL, Barnes GL. Improved survival of patients with cystic fibrosis. Med J Aust 1979;1:261-3.

8 Doull IJ. Recent advances in cystic fibrosis. Arch Dis Child 2001;85:62-6.

9 McCallum TJ, Milunsky JM, Cunningham DL, et al. Fertility in men with cystic fibrosis: an update on current surgical practices and outcomes. Chest 2000; 118:1059-62

10 Stern RC, Doershuck CF, Drumm M. 3849 C-T mutation and disease severity in cystic fibrosis. Lancet 1995;346:274-6.

11 Dreyfus DH, Bethel R, Gelfand EW. Cystic fibrosis 3849 C-T mutation associated with severe pulmonary disease and male fertility. Am J Respir Crit Care Med 1996;153:858-60.

12 Rosenlund B, Sjoblom P, Dimitrakopoulos A, et al. Epididymal and testicular sperm injection in the treatment of obstructive azoospermia. Acta Obstet Gynecol Scand 1997;75:135-9.

13 Hames A, Beesley J, Nelson R. Cystic fibrosis: what do patients know, and what else would they like to know? Respir Med 1991;85:389-92.

14 Nolan T, Desmond K, Herlich R, et al. Knowledge of cystic fibrosis in patients and their parents. Pediatrics 1986:77:229-35.

15 Sawyer SM, Tully M-AM, Dovey ME, et al. Reproductive health in males with cystic fibrosis: knowledge, attitudes and experiences of patients and parents. Pediatr Pulmonol 1998;25:226-30.

16 Thickett KM, Stableforth DE, Davis RE, et al. Awareness of infertility in men with cystic fibrosis. Fertil Steril 2001;76:407-8.

17 Fair A, Griffiths K, Osman LM. Attitudes to fertility among adults with cystic fibrosis in Scotland. Thorax 2000;55:672-7.

18 Madge SL, Carr SB. Comment. Pediatr Pulmonol 1999;27:293.

19 Nixon GM, Glazner JA, Martin JM, et al. Female sexual health care in cystic fibrosis. Arch Dis Child 2003;88:265-6.

20 Hull SC, Kass NE. Adults with cystic fibrosis and (in)fertility: how has the health care system responded? J Androl 2000;21:809-13.

21 Sawyer SM, Tulley M-AM, Colin AA. Reproductive and sexual health in males with cystic fibrosis: a case for health professional education and training. J Adol Health $2001 ; 28: 36-40$.

22 Lyon A, Bilton D. Fertility issues in cystic fibrosis. Paediatr Respir Rev 2002;3:236-40.

23 Sawyer SM. Reproductive health in young people with cystic fibrosis. Curr Opin Pediatr 1995;7:376-80.

24 Nixon GM, Glazner J, Martin J, et al. Urinary incontinence in female adolescents with cystic fibrosis. Pediatrics 2002;110:e22. 